\title{
Novel assisted cough system based on simulating cough airflow dynamics
}

\author{
Shuai Ren ${ }^{1,2}$ - Jinglong $\mathrm{Niu}^{3} \cdot$ Maolin $\mathrm{Cai}^{1} \cdot$ Liming Hao $^{1} \cdot$ Yan Shi ${ }^{1} \cdot$ Weiqing $\mathrm{Xu}^{1} \cdot$ Zujin Luo $^{4}$
}

Received: 4 January 2021 / Accepted: 26 March 2021 / Published online: 19 April 2021

(c) Zhejiang University Press 2021

\begin{abstract}
Cough is a defensive behavior that protects the respiratory system from infection and clears airway secretions. Cough airflow dynamics have been analyzed by a variety of mathematical and experimental tools. In this paper, the cough airflow dynamics of 42 subjects were obtained and analyzed. An identification model based on piecewise Gauss function for cough airflow dynamics is proposed through the dimensionless method, which could achieve over $90 \%$ identification accuracy. Meanwhile, an assisted cough system based on pneumatic flow servo system is presented. The vacuum situation and feedback control are used to increase the simulated peak cough flow rate, which are important for airway secretion clearance and to avoid airway collapse, respectively. The simulated cough peak flow could reach $5 \mathrm{~L} / \mathrm{s}$ without the external assistance such as manual pressing, patient cooperation and other means. Finally, the backstepping control is developed to generate a simulated cough airflow that closely mimics the natural cough airflow of humans. The assisted cough system opens up wide opportunities of practical application in airway secretion clearance for critically ill patients with COVID 2019 and other pulmonary diseases.
\end{abstract}

\section{Graphic abstract}
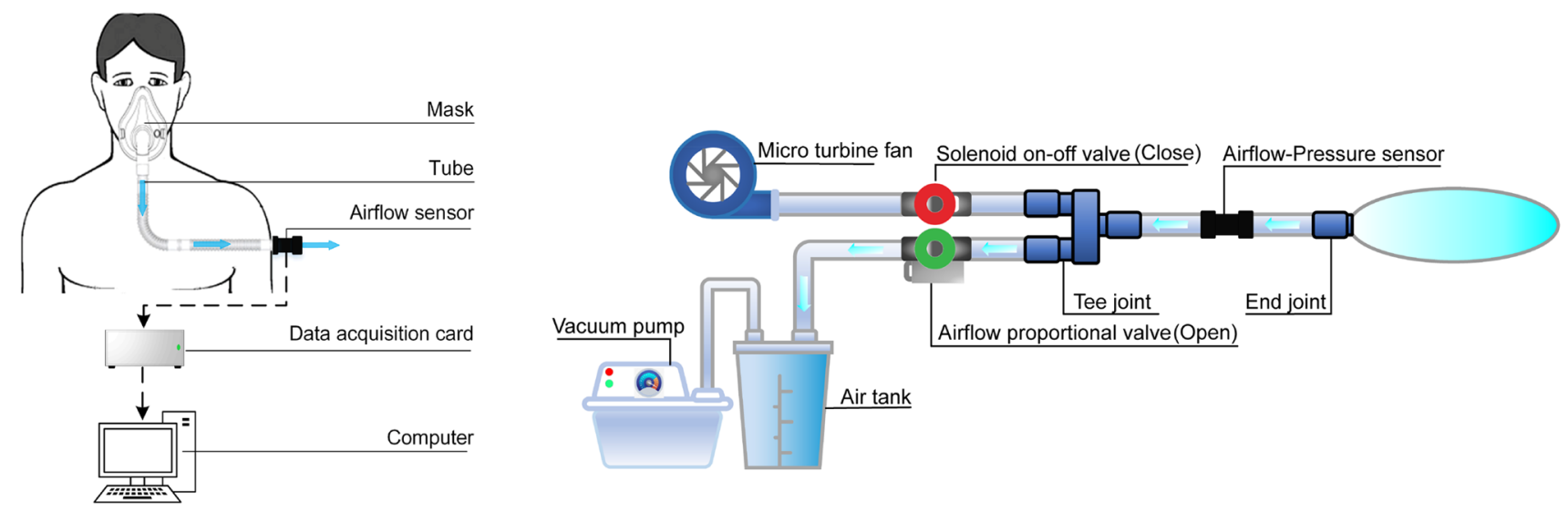

Keywords Airflow dynamics $\cdot$ Pneumatic system $\cdot$ Simulated cough $\cdot$ Model identification $\cdot$ Airway secretion clearance

Yan Shi

shiyan@buaa.edu.cn

$\triangle$ Zujin Luo

xmjg2002@163.com

1 School of Automation Science and Electrical Engineering, Beihang University, Beijing 100191, China

2 State Key Laboratory of Fluid Power and Mechatronic Systems, Zhejiang University, Hangzhou 310027, China
3 North Automatic Control Technology Institute, Taiyuan 030006, China

4 Department of Respiratory and Critical Care Medicine, Beijing Engineering Research Center of Respiratory and Critical Care Medicine, Beijing Institute of Respiratory Medicine, Beijing Chao-Yang Hospital, Capital Medical University, Beijing 100043, China 


\section{Introduction}

Cough is an irritable human reflex to clear foreign matter or particles from the respiratory tract to protect the respiratory system [1-3]. It is initiated with a deep inspiration to bring a large air volume into the lungs. Next, the larynx muscles close the glottis, and abdominal and thoracic muscles contract to generate high pressure in the lungs. Finally, the glottis suddenly opens and the expiratory muscles contract forcefully to release the air [4].

The cough process can be characterized by three key parameters: cough expired volume (CEV), peak velocity time (PVT) and cough peak flow rate (CPFR) [5-7]. These parameters can be used to analyze the airflow dynamics during the coughing process.

A lot of research has focused on the airflow dynamics during cough. Jennison used a high-speed camera to visualize the cough process [8]. The development of particle image velocimetry (PIV) technology has allowed many researchers to measure and capture the cough airflow information $[5,7,9,10]$. However, the acquisition intervals were too large to capture the cough airflow dynamics in sufficient detail. Gupta et al. used a spirometer to measure the cough airflow rate; results showed that the cough airflow rate variation over time can be represented as gamma probability distribution functions [11].

The CPFR variable is usually used to assess cough effectiveness and has a direct impact on airway clearance [12]. A normal individual may produce a CPFR as high as $12 \mathrm{~L} / \mathrm{s}$ [13]. However, for patients with respiratory muscle dysfunction such as neuromuscular disorders (NMDs) or emphysema, the secretion may not be cleared effectively due to weak or impaired cough [14]. Mechanical ventilation is commonly used in the intraoperative and postoperative management of critically ill patients [15]. For mechanical ventilated patients in intensive care units (ICUs), however, the cough may be impaired because of the endotracheal intubation hindering glottis closure, and also due to the frequent use of sedatives and muscle relaxants commonly used for mechanical ventilation synchrony. Secretion deposition in the airways caused by impaired cough may result in respiratory failure, hypoventilation, tracheobronchitis, pneumonia or other complications associated with secretion retention [16]. Therefore, the assisted cough device is essential for secretion clearance in patients with impaired cough.

In the early $1950 \mathrm{~s}$, Barach et al. proposed a physical method called "mechanical cough chamber" to simulate some of the mechanisms of the human cough $[17,18]$. The results of clinical observations in patients with poliomyelitis showed that the maximum expiratory flow rate reached an average $1.6 \mathrm{~L} / \mathrm{s}$, which was $145 \%$ of the most vigorous natural cough. Based on the development of noninvasive ventilation, the assisted cough device was later improved by using a blower to supply positive pressure for lung insufflation and negative pressure for rapid exsufflation, lately called mechanical insufflation exsufflation (MI-E). The MI-E supplies a maximum of $60 \mathrm{~cm} \mathrm{H}_{2} \mathrm{O}$-positive and negative pressure to generate a large peak cough flow that can exert a high shear force on secretions and propel them to the mouth. Bach et al. conducted some clinical researches on peak cough flow based on MI-E [19-21]. With patient cooperation, the peak cough flow could reach $6 \mathrm{~L} / \mathrm{s}$ or even higher. Recently, the MI-E has found numerous applications for secretion clearance in patients with chronic obstructive pulmonary disease (COPD), NMDs and other types of respiratory muscle dysfunction. Besides, MI-E is used for mechanical ventilated patients in ICU. As the most effective approach to simulate the natural cough, MI-E can propel bronchial secretions to the central airways where they cannot be cleared through tracheal suctioning [22]. Since most patients are unconscious and uncooperative with MI-E, the CPFR value is commonly less than $3 \mathrm{~L} / \mathrm{s}$, which reduces the effect of airway secretion clearance. Meanwhile, airway collapse may occur because of the large negative pressure produced at the exsufflation phase.

Our previous study proposed a simulated cough device based on a pneumatic pressure servo system featuring a maximal simulated cough peak flow that exceeds $4.2 \mathrm{~L} / \mathrm{s}$. Its cough clearance effectiveness has been verified through computational fluid dynamics simulation and experiments [23].

Herein, an identification model based on piecewise Gauss function for cough airflow dynamics is presented, and its identification accuracy is confirmed by experiments. In addition, a novel assisted cough system based on pneumatic flow servo system is proposed. In comparison with our previous study, the simulated cough peak flow produced by this system was further increased. In addition, feedback control is applied to avoid airway collapse. Finally, the backstepping control method is introduced into the system to simulate the natural human cough airflow dynamics. The assisted cough system and methods proposed in this study have promising value for secretion clearance in mechanically ventilated patients with impaired cough.

\section{Materials and methods}

\section{Cough airflow dynamics data collection and identification}

The variations in cough airflow rate with time were measured and recorded in a sitting position through a cough 
Fig. 1 The cough airflow rate collection system consists of a mask, a tube, an airflow sensor, a data acquisition card and a computer. The cough airflow collected by the mask flows through the tube and finally flows into the atmosphere through the airflow sensor
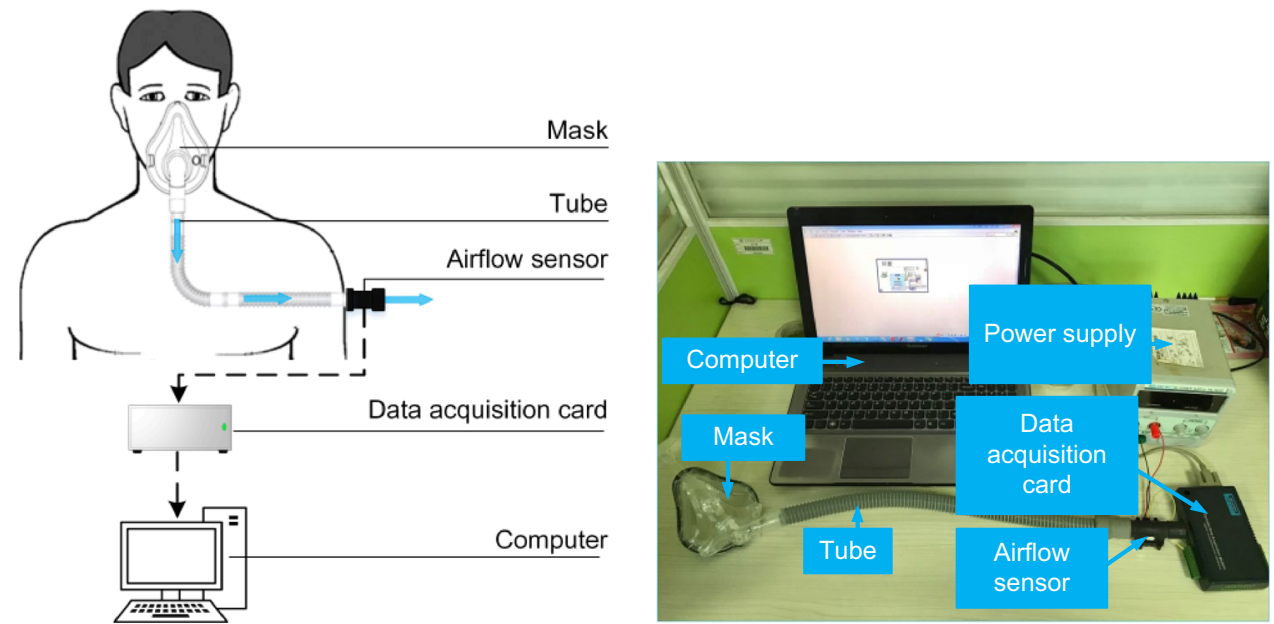

(a)

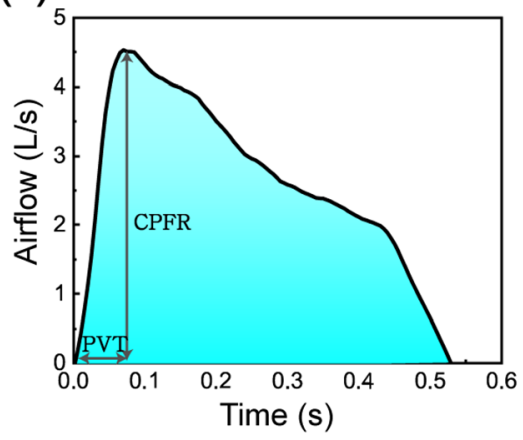

(b)

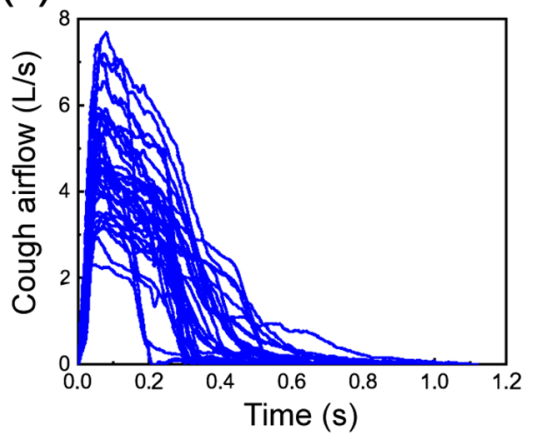

(c)

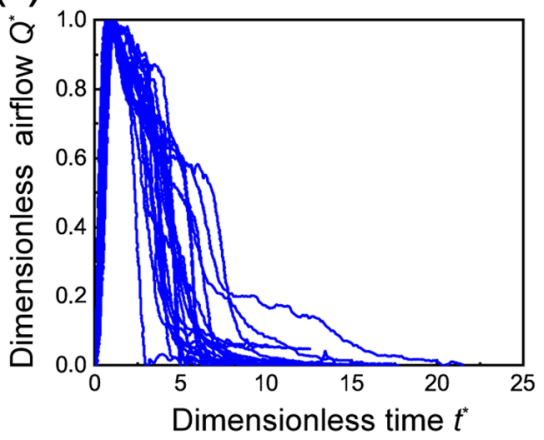

Fig. 2 The cough airflow dynamics. a An example of human cough airflow rate variation with time. The light blue color represents the cough expired volume during a cough process. b The cough airflow

airflow rate collection system in 42 healthy volunteers (18 females and 24 males), as shown in Fig. 1. All volunteers were instructed on how to produce a single forceful cough before data collection.

A ventilator mask and tube were employed to collect the airflow dynamics data. The tube connected the mask and the flow sensor. The flow sensor (FS6122, Siargo Ltd., Santa Clara, USA) with a frequency of $200 \mathrm{~Hz}$ was used to measure the cough flow rate variation over time. The acquired data were stored on an NI USB-6001 data acquisition device.

An example of cough airflow rate variation with time is presented in Fig. 2a. The CPFR and PVT are marked in the figure, and the light blue color represents the CEV. The cough airflow dynamics for all 42 subjects are illustrated in Fig. 2b, which indicates great differences among individuals. The variation ranges for CPFR, PVT and CEV are presented in Table 1. Considering the large variation ranges of cough airflow characteristics, the dimensionless method was used to analyze the cough airflow rates. The cough airflow rate and time were dimensionless by CPFR and PVT, rate variations of 42 healthy volunteers. $\mathbf{c}$ The dimensionless results of cough airflow rates

Table 1 Variation ranges of cough airflow characteristics for 42 volunteers

\begin{tabular}{lc}
\hline Parameter & Value range \\
\hline CPFR & $2.21-7.77 \mathrm{~L} / \mathrm{s}$ \\
PVT & $0.045-0.085 \mathrm{~s}$ \\
CEV & $0.34-2.52 \mathrm{~L}$
\end{tabular}

CPFR cough peak flow rate, $P V T$ peak velocity time, $C E V$ cough expired volume

respectively. The dimensionless airflow $Q^{*}$ and dimensionless time $t^{*}$ were calculated as

$$
\begin{aligned}
Q^{*} & =\frac{Q}{\mathrm{CPFR}}, \\
t^{*} & =\frac{t}{\mathrm{PVT}},
\end{aligned}
$$


where $Q$ and $t$ represent the cough airflow rate and time, respectively. The dimensionless results of cough airflow dynamics are shown in Fig. 2c.

Through observing variations in the dimensionless airflow, it is found that the curve variation trend coincides with the piecewise Gauss function. Therefore, an identification model for airflow dynamics based on piecewise Gauss function is proposed and presented as follows:

$Q^{*}= \begin{cases}a_{1} \cdot \mathrm{e}^{-\left(\frac{t^{*}-b_{1}}{c_{1}}\right)^{2},}, & t^{*} \leq b_{1}, \\ m+a_{2} \cdot \mathrm{e}^{-\left(\frac{t^{*}-b_{1}}{c_{2}}\right)^{2},}, & b_{1}<t^{*} \leq b_{2}, \\ a_{3} \cdot \mathrm{e}^{-\left(\frac{t^{*}-b_{2}}{c_{3}}\right)^{2},}, & t^{*}>b_{2},\end{cases}$

where $a_{1}, a_{2}, a_{3}, b_{1}, b_{2}, c_{1}, c_{2}, c_{3}$ and $m$ are the variables of the identification model. The MATLAB nonlinear least square curve fitting analysis function is used to obtain the optimum values of the variables of Eq. (3).

\section{The assisted cough system}

Considering that patients with impaired cough cannot inhale and exhale forcefully, a brushless direct current (DC) microturbine fan was used to supply the air into the lung and expand the lung volume. Meanwhile, a combination of vacuum pump and air tank was used to generate a near-vacuum situation for a high pressure difference. Figure 3 presents the whole assisted cough system. During the insufflation process shown in Fig. 3a, the solenoid on-off valve (VX234, SMC Ltd., Tokyo, Japan) opens, while the proportional valve (VEF2141,
SMC Ltd., Tokyo, Japan) closes. The microturbine fan supplies air with a certain pressure to the simulated lung, simulating the inhalation process during cough behavior. At the same time, the vacuum pump extracts the air from the air tank and generates a near-vacuum situation. The flow-pressure sensor (FS6122, Siargo Ltd., Santa Clara, USA) is used to measure the airflow and airway pressure. When the airway pressure reaches the insufflation setting value, the solenoid on-off valve closes, while the proportional valve opens. Due to the high pressure difference between the simulated lung and the air tank, air is expelled rapidly from the simulated lung to the air tank mimicking exhalation in natural cough behavior, as shown in Fig. 3b. The simulated cough airflow can be regulated by the proportional valve. The end exsufflation pressure is set and used to avoid the airway collapse during the exsufflation process. When the airway pressure reaches the end exsufflation pressure in the exsufflation process, the proportional valve closes and a single simulated cough cycle ends.

\section{The mathematical model of the assisted cough system}

In the insufflation process, the pressure supply mode was used for the microturbine fan; thus, the output pressure was as follows:

$p_{\mathrm{f}}= \begin{cases}\frac{p_{\text {set }}}{t_{\mathrm{r}}}, & t \leq t_{\mathrm{r}}, \\ p_{\mathrm{set}} & t>t_{\mathrm{r}},\end{cases}$

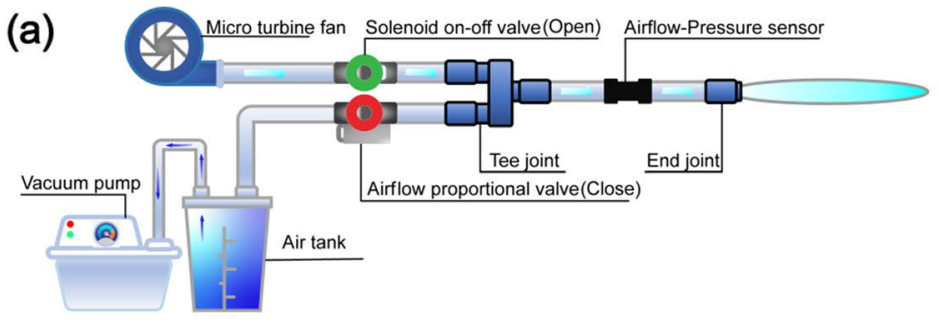

(c)
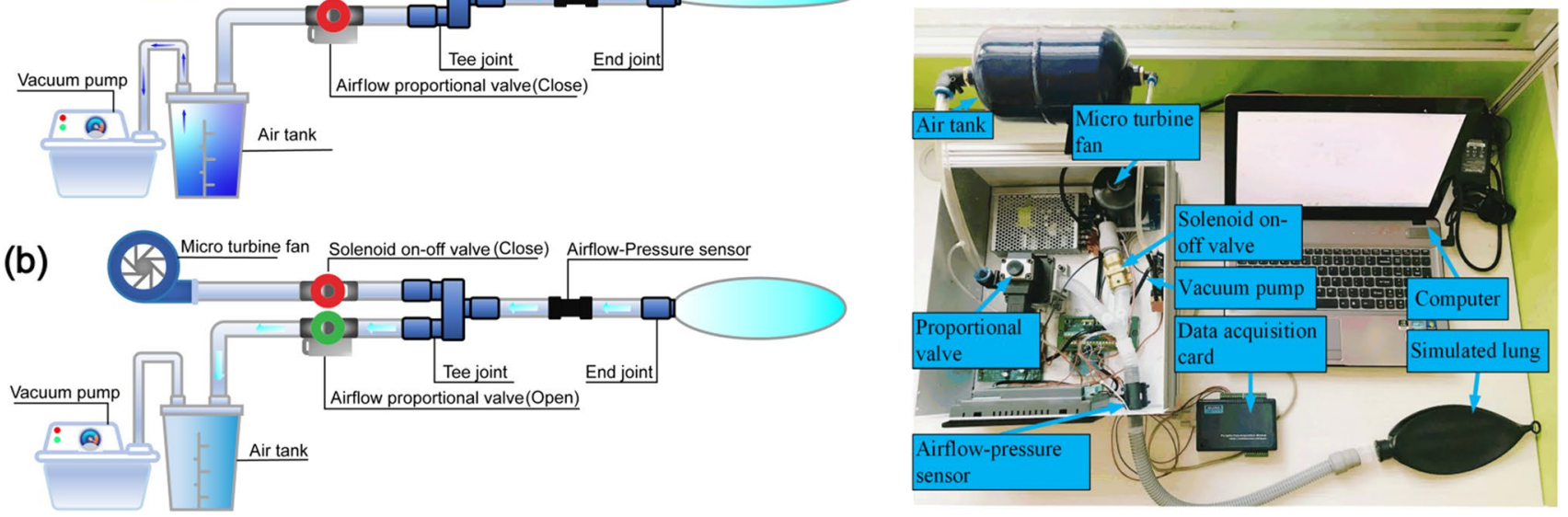

Fig. 3 The assisted cough system. a Insufflation process during cough behavior simulation. The solenoid on-off valve opens, while the proportional valve closes. The air supplied by the microturbine fan flows through solenoid on-off valve, tee joint, airflow-pressure sensor, end joint and finally into the simulated lung. b Exsufflation process dur- ing the simulation of natural cough behavior. The solenoid on-off valve closes, while the proportional valve opens. The air in the simulated lung flows through the end joint, airflow-pressure sensor, tee joint, airflow proportional valve and finally into the air tank. c The experimental system 
Table 2 Gas parameters under standard atmospheric pressure

\begin{tabular}{ll}
\hline Parameter & Value \\
\hline Temperature $\left({ }^{\circ} \mathrm{C}\right)$ & 20 \\
Dynamic viscosity $(\mathrm{Pa} \cdot \mathrm{s})$ & $18.1 \times 10^{-6}$ \\
Absolute pressure $(\mathrm{kPa})$ & 100 \\
Density $\left(\mathrm{kg} / \mathrm{m}^{3}\right)$ & 1.185 \\
Relative humidity & $65 \%$ \\
\hline
\end{tabular}

where $p_{\mathrm{f}}$ is the output pressure of the microturbine fan, $p_{\text {set }}$ denotes the insufflation setting pressure, and $t_{\mathrm{r}}$ is the pressure rise time.

The whole system works at standard atmospheric pressure. The gas parameters under standard atmospheric pressure are shown in Table 2. According to ISO6358 [24, $25]$, the mass flow rate through the solenoid valve is represented as

$q= \begin{cases}\frac{A_{\text {in }} p_{\mathrm{f}} \sqrt{1-b}}{\sqrt{R T}}, & \frac{p_{\mathrm{a}}}{p_{\mathrm{f}}} \leq b, \\ \frac{A_{\mathrm{in}} p_{\mathrm{f}} \sqrt{1-b}}{\sqrt{R T}} \sqrt{1-\left(\frac{p_{\mathrm{a}} / p_{\mathrm{f}}-b}{1-b}\right)^{2}}, & \frac{p_{\mathrm{a}}}{p_{\mathrm{f}}}>b,\end{cases}$

where $q$ is the gas mass flow rate, $A_{\text {in }}$ indicates the effective cross-sectional area of the solenoid valve, $R$ is the gas constant, $T$ stands for temperature, $p_{\mathrm{a}}$ is the pressure in the airway and the inlet pressure of the simulated lung, and $b$ is the critical pressure ratio.

This study uses the traditional circuit model for respiratory system [26, 27]. Respiratory compliance is regarded as the capacitance in the circuit system, respiratory resistance is regarded as the resistance in the circuit system, gas pressure is regarded as the voltage, and gas flow is regarded as the current. The model is presented as follows:

$p_{\mathrm{a}}=p_{1} \pm R_{\mathrm{r}} \cdot Q$.

The plus sign represents the insufflation process, while the minus sign represents the exsufflation process; $R_{\mathrm{r}}$ is the respiratory resistance, $Q$ indicates gas volume flow, and $p_{1}$ is the gas pressure in the simulated lung, which can be expressed as

$p_{1}=\frac{V_{1}}{C}$,

where $V_{1}$ is the air volume in the simulated lung, and $C$ is the compliance.

The gas mass flow rate has been commonly used to calculate the mathematical model of the solenoid valve. The conversion relationship between gas volume flow and mass flow is thus described as follows:
$Q=\frac{q}{\rho_{\mathrm{a}}}$,

where $\rho_{\mathrm{a}}$ indicates gas density.

Similarly to Eq. (5), the mass flow rate through the proportional valve is as follows:

$q= \begin{cases}\frac{A_{\mathrm{ex}} p_{\mathrm{a}} \sqrt{1-b}}{\sqrt{R T}}, & \frac{p_{\mathrm{t}}}{p_{\mathrm{a}}} \leq b, \\ \frac{A_{\mathrm{ex}} p_{\mathrm{a}} \sqrt{1-b}}{\sqrt{R T}} \sqrt{1-\left(\frac{p_{\mathrm{t}} / p_{\mathrm{a}}-b}{1-b}\right)^{2}}, & \frac{p_{\mathrm{t}}}{p_{\mathrm{a}}}>b,\end{cases}$

where $A_{\text {ex }}$ is the effective cross-sectional area of the proportional valve and can be regulated to generate different airflows; $p_{\mathrm{t}}$ denotes the pressure in the air tank.

The pressure change in the air tank is as follows:

$p_{\mathrm{t}}=p_{\text {dead }}+\frac{V_{\mathrm{e}} \cdot p_{\mathrm{atm}}}{V_{\mathrm{t}}}$,

where $p_{\text {dead }}$ is the dead space pressure caused by the vacuum pump, $p_{\text {atm }}$ denotes the atmospheric pressure, $V_{\mathrm{t}}$ is the volume of the air tank, $V_{\mathrm{e}}$ indicates the air volume which flows into the air tank and can be expressed as

$V_{\mathrm{e}}=\int Q \mathrm{~d} t$.

The MATLAB Simulink software was used for mathematical model simulation.

The control diagram and logic are presented in Fig. 4 and Eq. (12), respectively:

$\left\{\begin{array}{c}\left.\text { Insufflation (If } p_{\mathrm{a}}<p_{\mathrm{f}} \text { and } f<0\right), \\ \text { Exhalation }\left(\text { If } p_{\mathrm{a}}=p_{\mathrm{f}}\right), \\ \left.\text { Stop (If } p_{\mathrm{a}}<p_{\text {end }} \quad \text { and } f>0\right),\end{array}\right.$

where $f$ is the airflow, and $p_{\text {end }}$ is the end exsufflation pressure.

\section{The backstepping control method}

In this study, the backstepping control method was used for regulating the effective cross-sectional areas of the proportional valve during the cough process in order to generate a simulated cough airflow that resembles the natural human cough airflow more closely. Only the exsufflation process is considered in this section. The target airflow

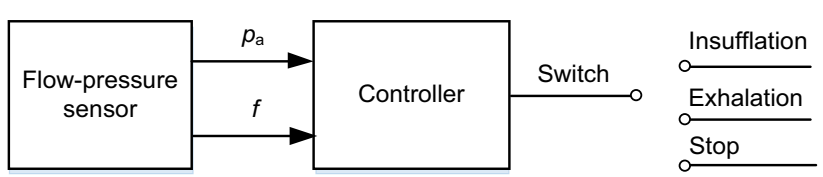

Fig. 4 Control diagram of the assisted cough system 
was generated based on the identification model for airflow dynamics, as previously established in this study. The detailed expression and deduction of control law for $A_{\text {ex }}$ are presented below.

If the state variable is set as $x=V=\int Q \mathrm{~d} t$, then $\dot{x}=Q$. The control object can be simplified to

$$
\begin{aligned}
\dot{x} & =\frac{A_{\mathrm{ex}} p_{\mathrm{a}} \sqrt{1-b}}{\rho_{\mathrm{a}} \sqrt{R T}} \sqrt{1-\left(\frac{p_{\mathrm{t}} / p_{\mathrm{a}}-b}{1-b}\right)^{2}} \\
& =\frac{A_{\mathrm{ex}}}{\rho_{\mathrm{a}} \sqrt{R T}} \sqrt{\frac{\left(p_{\mathrm{a}}+p_{\mathrm{t}}-2 b \cdot p_{\mathrm{a}}\right)\left(p_{\mathrm{a}}-p_{\mathrm{t}}\right)}{1-b}} .
\end{aligned}
$$

The effective cross-sectional area of the proportional valve $A_{\text {ex }}$ is the control variable of the system. If $v=\sqrt{\frac{\left(p_{\mathrm{a}}+p_{\mathrm{t}}-2 b \cdot p_{\mathrm{a}}\right)\left(p_{\mathrm{a}}-p_{\mathrm{t}}\right)}{1-b}}, \alpha=\frac{A_{\mathrm{ex}}}{\rho_{\mathrm{a}} \sqrt{R T}}$, then the expression of the controlled object state space will be

$\dot{x}=\alpha \cdot v$.

The Lyapunov energy function is presented as

$L=\frac{1}{2}\left(x-x_{\mathrm{d}}\right)^{2}$,

where $x_{\mathrm{d}}$ represents the expected value of the state, and $\dot{x}_{\mathrm{d}}$ represents the derivative of the expected value.

The differential form is

$$
\begin{aligned}
\dot{L} & =\left(x-x_{\mathrm{d}}\right)\left(\dot{x}-\dot{x}_{\mathrm{d}}\right) \\
& =\Delta x \cdot\left(\dot{x}-\dot{x}_{\mathrm{d}}\right) \\
& =\Delta x \cdot\left(\alpha \cdot v-\dot{x}_{\mathrm{d}}\right),
\end{aligned}
$$

where $\Delta x$ represents the difference between the actual state and the expected state, and $\Delta x=x-x_{\mathrm{d}}$.

The system control law can be set as $v=\frac{\dot{x}_{d}}{\alpha}-\Delta x$; thus, we can obtain:

$$
\begin{aligned}
\dot{L} & =\Delta x \cdot\left(\alpha \cdot v-\dot{x}_{\mathrm{d}}\right) \\
& =\Delta x \cdot\left(\dot{x}_{\mathrm{d}}-\alpha \cdot \Delta x-\dot{x}_{\mathrm{d}}\right) \\
& =-\alpha \cdot \Delta x^{2}<0 .
\end{aligned}
$$

It can be seen that the first derivative of the Lyapunov energy function presents as negative, which verifies the stability of the system.

The system control variable $\left(A_{\mathrm{ex}}\right)$ can be calculated as

$$
\begin{aligned}
& \sqrt{\frac{\left(p_{\mathrm{a}}+p_{\mathrm{t}}-2 b \cdot p_{\mathrm{a}}\right)\left(p_{\mathrm{a}}-p_{\mathrm{t}}\right)}{1-b}}+\Delta x=\frac{\dot{x}_{\mathrm{d}}}{\alpha}, \\
& \alpha=\frac{\dot{x}_{\mathrm{d}}}{\sqrt{\frac{\left(p_{\mathrm{a}}+p_{\mathrm{t}}-2 b \cdot p_{\mathrm{a}}\right)\left(p_{\mathrm{a}}-p_{\mathrm{t}}\right)}{1-b}}+\Delta x}, \\
& A_{\mathrm{ex}}=\frac{\dot{x}_{\mathrm{d}} \cdot \rho_{\mathrm{a}} \sqrt{R T}}{\sqrt{\frac{\left(p_{\mathrm{a}}+p_{\mathrm{t}}-2 b \cdot p_{\mathrm{a}}\right)\left(p_{\mathrm{a}}-p_{\mathrm{t}}\right)}{1-b}}+\Delta x},
\end{aligned}
$$

where $\dot{x}_{\mathrm{d}}$ is the target flow of the system, and $\Delta x$ indicates the integration of error of target flow and output flow.

\section{Results}

\section{Identification of human cough airflow dynamics}

Based on the MATLAB curve fitting analysis, the optimum values of the variables of Eq. (3) are presented as follows:

$$
\left\{\begin{array}{l}
\xi=\frac{\mathrm{CEV}}{\mathrm{CPFR} \times \mathrm{PVT}} \\
a_{1}=1 \\
b_{1}=1 \\
c_{1}=0.6018 \\
m=-0.0694 \cdot \xi+0.9755 \\
a_{2}=1-m \\
b_{2}=0.8714 \cdot \xi+0.501 \\
c_{2}=0.2765 \cdot \xi+0.1463 \\
a_{3}=m+a_{2} \cdot \mathrm{e}^{-\left(\frac{b_{2}-1}{c_{2}}\right)^{2}} \\
c_{3}=0.6292 \cdot \xi-0.9858
\end{array}\right.
$$

In order to prove the accuracy of the identification model, the cough airflow data of four new volunteers (two males and two females) with different cough airflow characteristics were collected. Their cough airflow characteristics are presented in Table 3, and Fig. 5 shows the respective identification results. All four cases have demonstrated that the identification model curves are in good agreement with the collected cough airflow curves.

Table 3 The cough airflow characteristics for four new volunteers

\begin{tabular}{llll}
\hline Volunteer & CPFR (L/s) & PVT $(\mathrm{s})$ & CEV $(\mathrm{L})$ \\
\hline $\mathrm{a}$ & 5.81 & 0.075 & 1.51 \\
$\mathrm{~b}$ & 2.98 & 0.055 & 0.58 \\
$\mathrm{c}$ & 6.59 & 0.070 & 1.82 \\
$\mathrm{~d}$ & 4.57 & 0.065 & 1.11 \\
\hline
\end{tabular}

$C P F R$ cough peak flow rate, $P V T$ peak velocity time, $C E V$ cough expired volume 
(a)

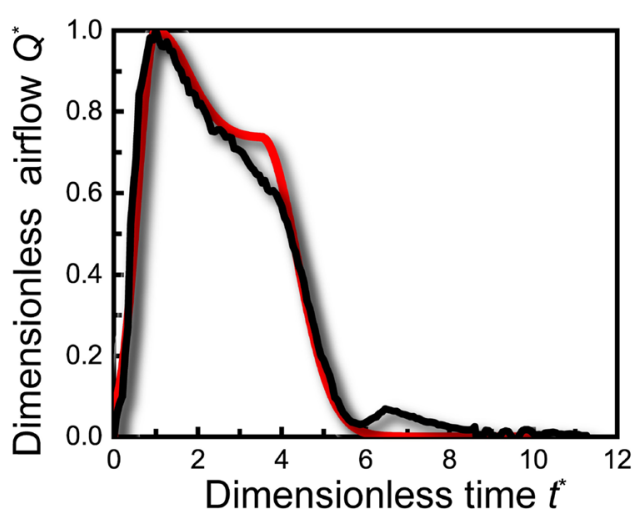

(c)

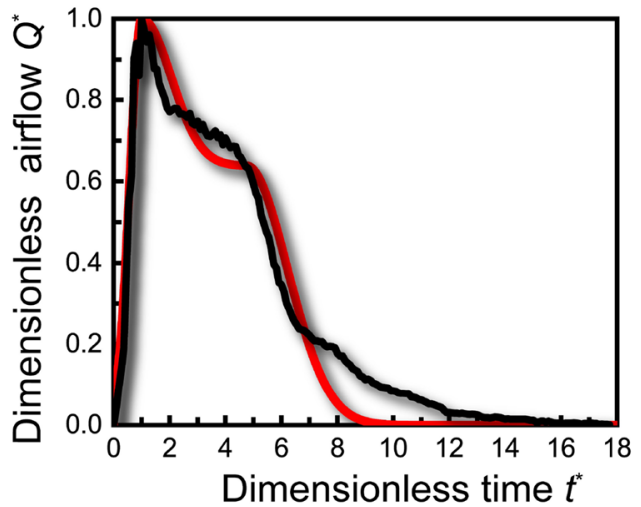

(b)

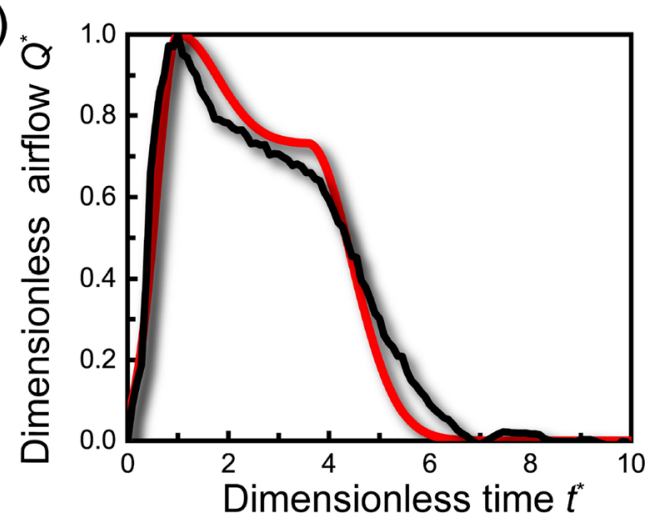

(d)

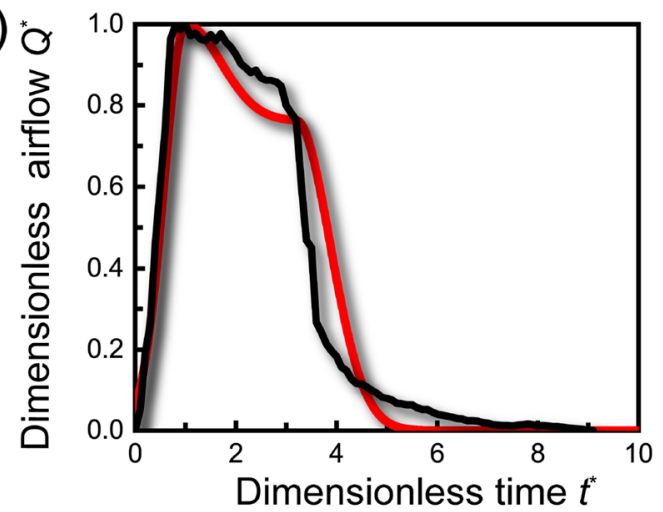

Fig. 5 Identification results for four new volunteers. The black lines represent the collected and dimensionless curves, and the red lines represent the identification model curves. a Volunteer a. b Volunteer b. c Volunteer c. d Volunteer d

Table $4 R$-square values of the identification model

\begin{tabular}{ll}
\hline Volunteer & $R$-square \\
\hline $\mathrm{a}$ & 0.9833 \\
$\mathrm{~b}$ & 0.9666 \\
$\mathrm{c}$ & 0.9582 \\
$\mathrm{~d}$ & 0.9398 \\
\hline
\end{tabular}

Additionally, $R$-square values were calculated to quantify the identification accuracy and are presented in Table 4 . The calculation of $R$-square is as follows:

$R^{2}=1-\frac{\mathrm{SSE}}{\mathrm{SST}}$

where SSE is the sum of squares of residuals, and SST is the sum of total squares. The calculation formulas for SSE and SST are performed as follows:

$\mathrm{SSE}=\sum_{i=1}^{n}\left(y_{i}-\hat{y}_{i}\right)^{2}$,
Table 5 Experimental setup parameters

$\mathrm{SST}=\sum_{i=1}^{n}\left(y_{i}-\bar{y}\right)^{2}$,

where $y_{i}$ indicates the measured value, $\hat{y}_{i}$ is the model fitting value, and $\bar{y}$ denotes the mean value.

\section{Airflow dynamics of the cough assisted system}

Figure $3 c$ shows the experimental system. The experimental and simulation studies were conducted to test the airflow 


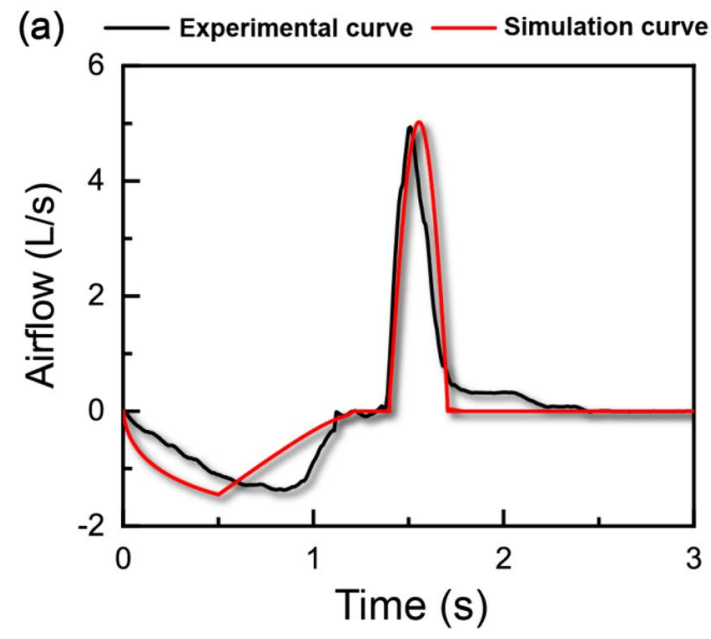

Fig. 6 The simulated cough airflow dynamics. a The airflow in a single simulated cough process. The negative airflow represents the insufflation process, while the positive airflow represents the exsuffla-

dynamics of the cough assisted system. The setup parameters used in the experiment and simulation are shown in Table 5, and the airflow and airway pressure during a single simulated cough process are presented in Fig. 6a and 6b, respectively.

According to the comparison of the simulation and experimental curves in Fig. 6, the simulation results were consistent with the experimental results, which confirms the accuracy of the mathematical model of the assisted cough system. It can be seen in Fig. 6a that the simulated cough peak flow reaches $5 \mathrm{~L} / \mathrm{s}$. Due to the feedback control, the

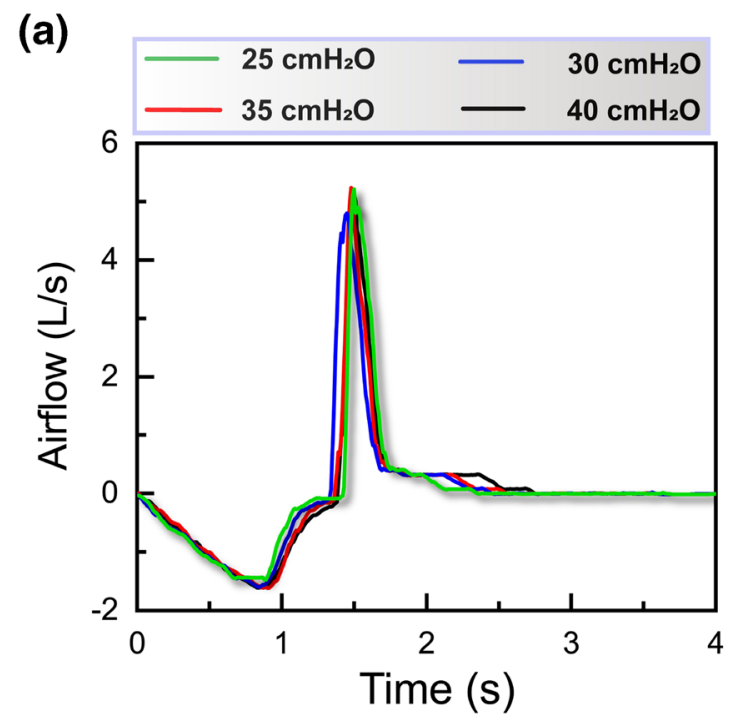

Fig. 7 The simulated cough airflow dynamics for different pressures and effective cross-sectional areas of the proportional valve $\left(A_{\mathrm{ex}}\right)$. a Airflows under four different insufflation setting pressures (1

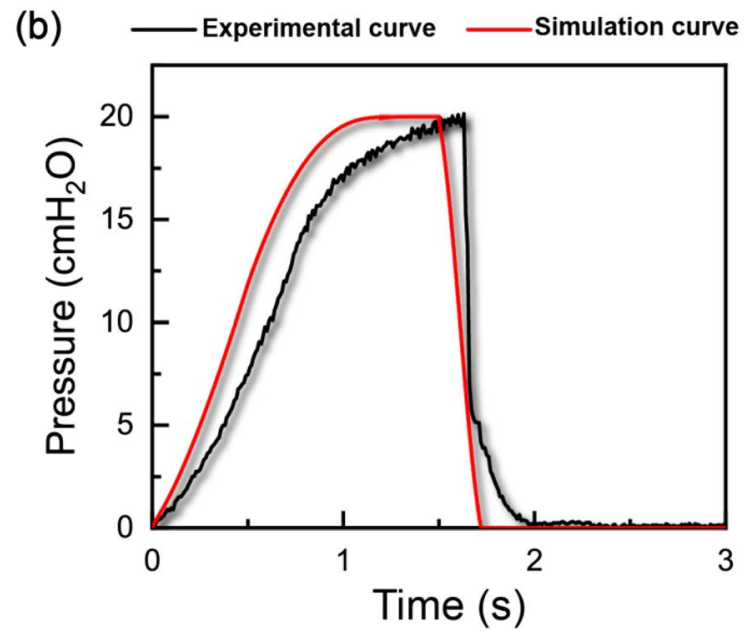

tion process. $\mathbf{b}$ The airway pressure in a single simulated cough process $\left(1 \mathrm{cmH}_{2} \mathrm{O}=98 \mathrm{~Pa}\right)$

pressure in the airway was always above the atmospheric pressure which avoiding airway collapse, as shown in Fig. 6b.

The influences of insufflation setting pressure and effective cross-sectional area of the proportional valve $\left(A_{\mathrm{ex}}\right)$ on simulated cough airflow dynamics were also analyzed. The simulated cough airflows under four different insufflation setting pressures are presented in Fig. 7a. The changes of peak flow rates of the simulated cough were not obvious along the increase in the insufflation setting pressure. Meanwhile, the simulated cough airflows under four different

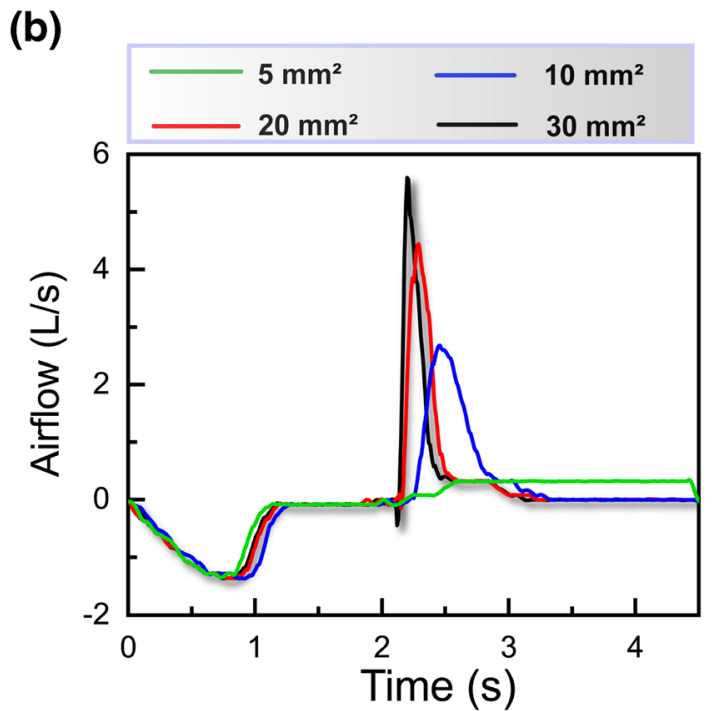

$\mathrm{cmH}_{2} \mathrm{O}=98 \mathrm{~Pa}$ ). b Airflows under four different effective cross-sectional areas of the proportional valve 
effective cross-sectional areas of the proportional valve are shown in Fig. 7b. The peak flow rate of simulated cough significantly increased with the growth of effective crosssectional area.

\section{Backstepping control method for simulating human cough}

It is established from Fig. 6a that the simulated cough airflow generated by the assisted cough system is a pulsed airflow that is different from the human cough airflow. In this study, the backstepping control method was used for regulating the effective cross-sectional areas of the

Table 6 The cough airflow characteristics of five subjects

\begin{tabular}{llll}
\hline Subject & CPFR (L/s) & PVT (s) & CEV (L) \\
\hline $\mathrm{a}$ & 3.27 & 0.060 & 0.83 \\
$\mathrm{~b}$ & 4.26 & 0.055 & 0.82 \\
$\mathrm{c}$ & 5.46 & 0.055 & 1.58 \\
$\mathrm{~d}$ & 6.59 & 0.070 & 1.75 \\
$\mathrm{e}$ & 7.77 & 0.085 & 2.27 \\
\hline
\end{tabular}

$C P F R$ cough peak flow rate, $P V T$ peak velocity time, $C E V$ cough expired volume proportional valve during the cough process in order to generate a simulated cough airflow that resembles the natural human cough airflow more closely. The target airflow was generated based on the identification model for airflow dynamics as previously established in this study, which is in good agreement with the natural human cough airflow. Five subjects were selected to generate the target airflows through the identification model. The cough airflow characteristics for these five subjects are presented in Table 6 .

The simulation results for simulated cough airflows based on the backstepping control method are presented in Fig. 8a-8e. Owing to the regulation of the $A_{\mathrm{ex}}$ of proportional valve during the simulated cough process, the simulated cough airflows tended to be close to the natural human cough airflow. Significant errors were only observed in the rising stages of the airflows; the maximum error and error rate were calculated and are presented in Fig. 8f, where the maximum error rate is the ratio of error and CPFR. As shown in the figure, the maximum error and error rate increased with the rise of CPFR in the overall presentation. The maximum error exceeded $2.5 \mathrm{~L} / \mathrm{s}$, and the maximum error rates were under $35 \%$ for all five subjects. (a)

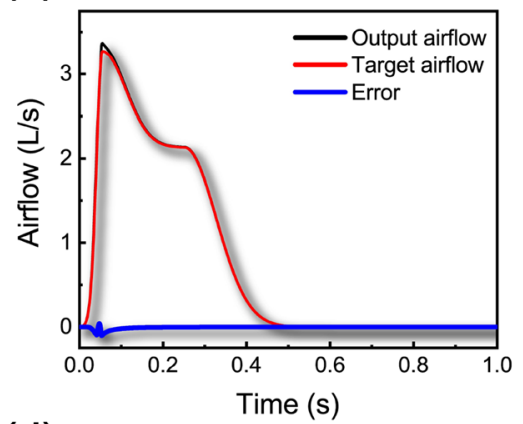

(d)

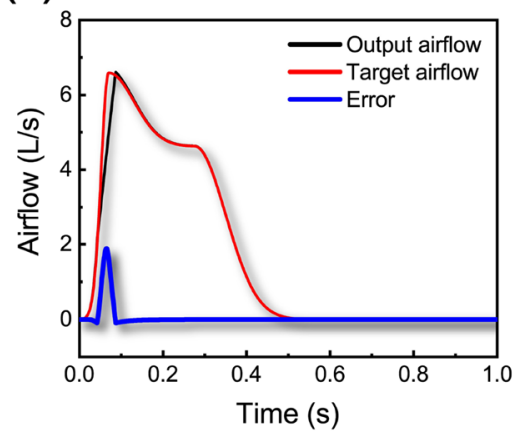

(b)

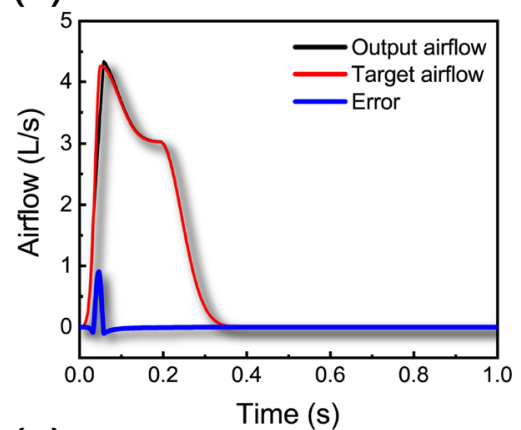

(e)

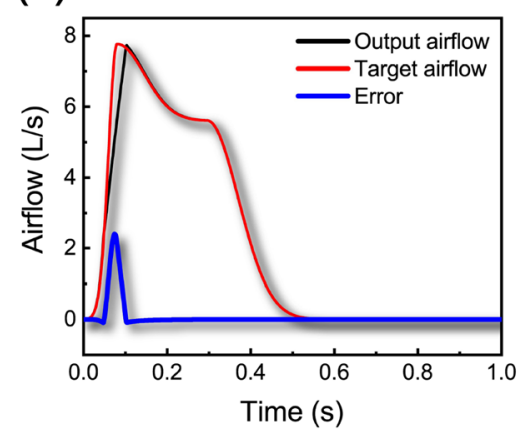

(c)

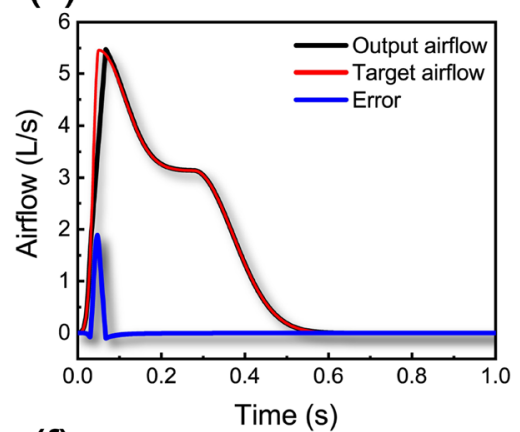

(f)

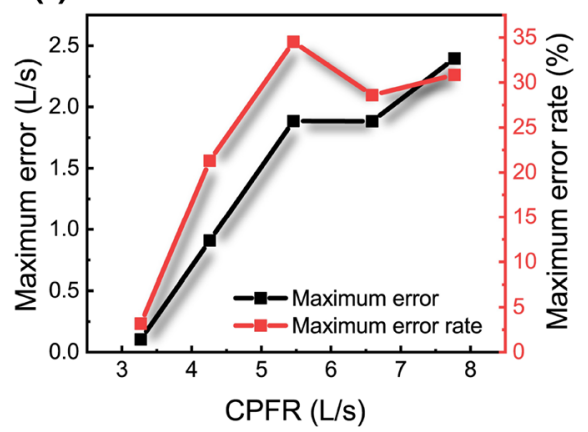

Fig. 8 Simulation results for simulated cough airflows based on the backstepping control method for subject a (a), subject b (b), subject c (c), subject d (d) and subject e (e). In a-e, the black curve (output airflow) is the airflow generated by the assisted cough system, the red curve (target airflow) is the identification model curve, and the blue curve is the error between the output airflow and target airflow. $f$ The error analysis of simulated cough airflows for all five subjects 


\section{Discussion}

A new identification model based on the piecewise Gauss function for cough airflow dynamics was proposed in this paper. The variables of identification model were represented by three key parameters, namely CEV, CPFR and PVT. The $R$-square values were calculated to quantify the identification accuracy. The closer the value is to 1 , the higher the identification accuracy is, and the closer it is to 0 , the lower the identification accuracy. Table 4 shows the $R$-square values of the four volunteers, which are all above 0.9 ; thus, the accuracy of identification model based on the piecewise Gauss function for cough airflow dynamics is over $90 \%$. Therefore, this model provides an appropriate and accurate method to describe the human cough airflow dynamics. Given that the CPFR, PVT and CEV values of a person are obtained, their characteristic cough airflow dynamics can be predicted. Moreover, the identification model can be used as boundary conditions for a finite element analysis of gas-secretion interaction during cough behavior.

Furthermore, an assisted cough system based on pneumatic flow servo system was proposed. The feedback control method was described and used to avoid airway collapse. A vacuum situation was generated and applied to increase the simulated peak cough flow rate that is of major importance for airway secretion clearance. In order to prevent hyperventilation, the insufflation pressure range must be $0-40$ $\mathrm{cmH}_{2} \mathrm{O}$, which is consistent with the ventilator setting. Based on the experimental results shown in Fig. 7a and 7b, it is considered that the effective cross-sectional area of the proportional valve has a higher impact on simulated cough airflow than insufflation pressure. The simulated cough peak flow can reach $5 \mathrm{~L} / \mathrm{s}$, which exceeds the successful extubation and provides the necessary airflow to effectively clear secretions $(2.67 \mathrm{~L} / \mathrm{s})$ [20]. Because of the vacuum feature, the assisted cough system proposed in this study can produce the largest possible simulated peak cough flow in the absence of external assistance such as manual pressing, patient cooperation and other means. However, because of the limitation of effective cross-sectional area of the proportional valve, the simulated peak cough flow was still lower than that of natural human cough that can produce a peak flow rate as great as $12 \mathrm{~L} / \mathrm{s}$. An effective solution for further improving the simulated peak cough flow is the combination of functional electrical stimulation; such an assisted cough system is our proposed follow-up research content. The functional electrical stimulation of abdominal muscles can make the muscles contract and generate high pressure in the lungs, thus improving the driving pressure for a cough. Therefore, the assisted cough system opens up opportunities for wide practical application in airway secretion clearance for mechanical ventilation patients with impaired cough.
In addition, the backstepping control method was developed to generate a simulated cough airflow that is close to the natural human cough airflow. Figure $8 \mathrm{a}-8 \mathrm{e}$ demonstrates that the output airflows lag behind the target airflows. The hysteresis becomes more obvious with increasing CPFR, which is caused by the response time and maximum effective cross-sectional area of the proportional valve (their values are $20 \mathrm{~ms}$ and $45 \mathrm{~mm}^{2}$, respectively). When the CPFR of target airflow increases to a higher value, shorter response time and higher effective cross-sectional area are needed to prevent hysteresis. The backstepping control method applied to the assisted cough system can be used to increase man-machine coordination during airway secretion clearance and to realize the "simulation of natural cough airflow" in a true sense.

\section{Conclusions}

In this paper, the cough airflow dynamics of 42 subjects were analyzed, and a new identification model based on piecewise Gauss function for cough airflow dynamics was proposed, whose identification accuracy could achieve over $90 \%$. This model could provide an appropriate and accurate method to describe the human cough airflow dynamics. Meanwhile, an assisted cough system based on pneumatic flow servo system was presented, and its mathematical model was verified through experiments. Vacuum and feedback control were used to increase the simulated peak cough flow rate and to avoid the airway collapse, respectively. The simulated cough peak flow could reach $5 \mathrm{~L} / \mathrm{s}$ without external assistance. The airway pressure remained above atmospheric pressure during the entire process, and airway collapse was avoided. Furthermore, the backstepping control method was developed to generate a simulated cough airflow that is closer to the natural human cough airflow.

The proposed assisted cough system is expected to provide wide practical applications in airway secretion clearance for critically ill patients with COVID 2019 and other pulmonary diseases.

Acknowledgements The research is supported by the National Natural Science Foundation of China (No. 52005015), the China Postdoctoral Science Foundation (No. 2019M660391), the Open Foundation of the State Key Laboratory of Fluid Power and Mechatronic Systems (No. GZKF-201920), the Outstanding Young Scientists in Beijing (No. BJJWZYJH01201910006021) and the National Key Research and Development Project (No. 2019YFC0121702).

Author contributions SR and JLN designed the cough airflow collection system and developed the cough airflow dynamics model. SR designed the assisted cough system. SR and LMH conducted the experiments and wrote the manuscript. MLC, YS and WQX supervised the experiments and provided advice on the system design and data 
analysis. YS and MLC performed critical revision of the manuscript. $\mathrm{ZJL}$ and YS assisted in the data collection.

\section{Declarations}

Conflict of interest The authors declare that there is no conflict of interest.

Ethical approval The cough airflow dynamics data of 42 human subjects were obtained by the doctors at the Beijing Chao-Yang Hospital. All the human subjects were agreed to conduct these measurements and signed the informed content. The Beijing Chao-Yang Hospital Ethics Committee and Human Subjects have approved these data collections (Approval number: 20175241). All procedures followed were in accordance with the ethical standards of the responsible committee on human experimentation (institutional and national) and with the Helsinki Declaration of 1975, as revised in 2008 (5).

\section{References}

1. Dubuis E, Wortley MA, Grace MS et al (2014) Theophylline inhibits the cough reflex through a novel mechanism of action. J Allergy Clin Immunol 133(6):1588-1598. https://doi.org/10. 1016/j.jaci.2013.11.017

2. Laghi F, Maddipati V, Schnell T et al (2017) Determinants of cough effectiveness in patients with respiratory muscle weakness. Respir Physiol Neurobiol 240:17-25. https://doi.org/10.1016/j. resp.2017.02.005

3. Zhang B, Zhu C, Ji ZM et al (2017) Design and characterization of a cough simulator. J Breath Res 11(1):016014. https://doi.org/ 10.1088/1752-7163/aa5cc6

4. Fink JB (2007) Forced expiratory technique, directed cough, and autogenic drainage. Respir Care 52(9):1210-1221

5. Mahajan RP, Singh P, Murty GE et al (1994) Relationship between expired lung volume, peak flow rate and peak velocity time during a voluntary cough manoeuvre. Br J Anaesth 72(3):298-301. https://doi.org/10.1093/bja/72.3.298

6. Singh P, Mahajan RP, Murty GE et al (1995) Relationship of peak flow rate and peak velocity time during voluntarv couzhinc. Br J Anaesth 74(6):714-716. https://doi.org/10.1093/bja/74.6.714

7. Vansciver M, Hertzberg J, Miller S et al (2011) Particle image velocimetry of human cough. AS\&T J 45(3):415-422. https://doi. org/10.1080/02786826.2010.542785

8. Jennison MW (1941) Atomizing of mouth and nose secretions into the air as revealed by high-speed photography. In: Aerobiology (Pub. No. 17). American Association for the Advancement of Science, Washington, DC, pp 106-128

9. Chao CYH, Wan MP, Morawska L et al (2009) Characterization of expiration air jets and droplet size distributions immediately at the mouth opening. J Aerosol Sci 40(2):122-133. https://doi.org/ 10.1016/j.jaerosci.2008.10.003

10. Kwon SB, Park J, Jang J et al (2012) Study on the initial velocity distribution of exhaled air from coughing and speaking. Chemosphere 87(11):1260-1264. https://doi.org/10.1016/j.chemosphere. 2012.01.032

11. Gupta JK, Lin CH, Chen Q et al (2010) Flow dynamics and characterization of a cough. Indoor Air 19(6):517-525. https://doi.org/ 10.1111/j.1600-0668.2009.00619.x
12. Bach J (1996) Criteria for extubation and tracheostomy tube removal for patients with ventilator failure. Chest 110:1566-1571. https://doi.org/10.1378/chest.110.6.1566

13. Leiner GC, Abromowitz S, Small MJ et al (1963) Expiratory peak flow rate. standard values for normal subjects. Am Rev Respir Dis 88:644-651

14. Burle JF, Vincent B, Lachal R et al (2018) Mechanical insufflation-exsufflation: room for improvement. Respir Care 63(10):1318-1319. https://doi.org/10.4187/respcare.06542

15. Kwong MT, Colopy GW, Weber AM et al (2019) The efficacy and effectiveness of machine learning for weaning in mechanically ventilated patients at the intensive care unit: a systematic review. Bio-Des Manuf 2(1):31-40. https://doi.org/10.1007/ s42242-018-0030-1

16. de Camillis Márcio Luiz F, Augusto S, Goulart Rosa R et al (2018) Effects of mechanical insufflation-exsufflation on airway mucus clearance among mechanically ventilated ICU subjects. Respir Care 63(12):1471-1477. https://doi.org/10.4187/respcare.06253

17. Bickerman HA, Beck GJ, Gordon C et al (1952) Physical methods simulating mechanisms of the human cough. J Appl Physiol 5(2):85-91. https://doi.org/10.1152/jappl.1952.5.2.85

18. Barach AL, Beck GJ, Bickerman HA et al (1952) Physical methods simulating cough mechanisms: use in poliomyelitis, bronchial asthma, pulmonary emphysema, and bronchiectasis. J Am Med Assoc 150(14):1380-1385

19. Bach JR (1993) Mechanical insufflation-exsufflation. Comparison of peak expiratory flows with manually assisted and unassisted coughing techniques. Chest 104(5):1553-1562. https://doi.org/10. 1378/chest.104.5.1553

20. Bach JR (1995) Amyotrophic lateral sclerosis: predictors for prolongation of life by noninvasive respiratory aids. Arch Phys Med Rehabil 76(9):828-832. https://doi.org/10.1016/s0003-9993(95) 80547-8

21. Bach JR, Ishikawa Y, Kim H (1997) Prevention of pulmonary morbidity for patients with Duchenne muscular dystrophy. Chest 112(4):1024-1028. https://doi.org/10.1378/chest.112.4.1024

22. Terzi N, Prigent H, Lofaso F et al (2018) Mechanical insufflationexsufflation to improve secretion clearance during invasive ventilation. Respir Care 63(12):1577-1578. https://doi.org/10.4187/ respcare.06700

23. Ren S, Shi Y, Cai M et al (2018) Ansys-matlab co-simulation of mucus flow distribution and clearance effectiveness of a new simulated cough device. Int J Numer Method Biomed Eng 34(6):e2978. https://doi.org/10.1002/cnm.2978

24. Ren S, Cai M, Shi Y et al (2017) Influence of bronchial diameter change on the airflow dynamics based on a pressure-controlled ventilation system. Int J Numer Method Biomed Eng 34(3):e2929. https://doi.org/10.1002/cnm.2929

25. Ren S, Shi Y, Cai M et al (2018) Influence of airway secretion on airflow dynamics of mechanical ventilated respiratory system. IEEE/ACM Trans Comput Biol Bioinform 15(5):1660-1668. https://doi.org/10.1109/TCBB.2017.2737621

26. Rideout VC (1991) Mathematical and computer modeling of physiological systems. Prentice-Hall

27. D'Angelo E, Calderini E, Torri G et al (1989) Respiratory mechanics in anesthetized paralyzed humans: effects of flow, volume, and time. J Appl Physiol 67(6):2556-2564 